Net abol i c syndrome is not a predi ct or for car di ovascul ar events in Japanese pati ents wi th di abet es mel I it us asympt omat i c for cor onary artery di sease: A r et rospective anal ysi s of the J-ACCESS 2 st udy

\begin{tabular}{|l|l|}
\hline 著者 & $\begin{array}{l}\text { Nakaj i ma Keni chi , Takei shi Yasuchi ka, Nat suo } \\
\text { Shi nr o, Yamasaki Yoshi mi t su, N shi mur a } \\
\text { Tsunehi ko }\end{array}$ \\
\hline $\begin{array}{l}\text { j our nal or } \\
\text { publ i cat i on ti tl e }\end{array}$ & Journal of Nucl ear Car di ol ogy \\
\hline vol une & 20 \\
\hline number & 2 \\
\hline page range & 234 241 \\
\hline year & 2013 04 01 \\
\hline URL & ht t p: //hdl . handl e. net /2297/33408 \\
\hline
\end{tabular}




\title{
Metabolic syndrome is not a predictor for cardiovascular events in Japanese patients with diabetes mellitus asymptomatic for coronary artery disease: A retrospective analysis of the J-ACCESS-2 study
}

\author{
Kenichi Nakajima (1), Yasuchika Takeishi (2), Shinro Matsuo (1), Yoshimitsu Yamasaki (3), Tsunehiko \\ Nishimura (4) \\ 1) Department of Nuclear Medicine, Kanazawa University Hospital, Kanazawa, Japan \\ 2) Department of Cardiology and Hematology, Fukushima Medical University, Fukushima, Japan \\ 3) Department of Endocrinology and Metabolism, Osaka University, Suita, Japan \\ 4) Department of Radiology, Kyoto Prefectural University of Medicine, Kyoto, Japan
}

\section{Correspondence:}

Kenichi Nakajima, MD, Department of Nuclear Medicine, Kanazawa University Hospital 13-1 Takara-machi, Kanazawa, 920-8641, Email: nakajima@med.kanazawa-u.ac.jp and Tsunehiko Nishimura, MD, Department of Radiology, Kyoto Prefectural University of Medicine, Kyoto, Japan,nisimura@koto.kpu-m.ac.jp

\begin{abstract}
Purpose. Patients with metabolic syndrome (MetS) have potentially higher risk for cardiovascular events. The aim of this study was to evaluate the effect of MetS on cardiac events in type-2 diabetic patients asymptomatic for coronary artery disease (CAD) in a Japanese population.

Methods. A total of 485 patients from a J-ACCESS-2 investigation with stress gated myocardial perfusion imaging (MPI) and quantitative gated MPI analysis (QGS) were examined. Cardiovascular hard events (cardiac death and acute coronary syndrome) and total events during a three-year follow-up were analyzed.

Results . The MetS group $(n=229)$ had higher incidence of hypertension, dyslipidemia and ventricular dilatation than the non-MetS group $(n=256)$. The hard events were 8 and 12 for the MetS and non-MetS groups ( $\mathrm{p}=\mathrm{n}$. s.), and total events were 31 and 31 for each of these groups, respectively $(\mathrm{p}=\mathrm{n} . \mathrm{s}$.). Significant variables related to total cardiovascular events included age, current smoking, insulin use, total cholesterol, ejection fration, summed stress score $\geq 9$ and summed difference score $\geq 2$. Cox proportional hazard analysis and Kaplan-Meier survival analysis showed that only the summed stress score was related to total events $(\mathrm{p}=0.01)$, and the presence and number of items for MetS criteria were not.

Conclusion. In patients with type-2 diabetes asymptomatic for CAD, cardiovascular events and ischemia are as common in diabetic patients without MetS as in those with MetS. A high MPI defect score is related to total events including cardiac and cerebrovascular events.
\end{abstract}

Key words: metabolic syndrome, type-2 diabetes, myocardial perfusion imaging, multi-center study, prognosis

\section{Introduction}

Metabolic syndrome (MetS) is defined by the combinations of diabetes mellitus, hypertension, dyslipidemia, central obesity and microalbuminuria by the World Health Organization and National Cholesterol Education Program Adult Treatment Panel III, and it is considered an important risk factor for future cardiovascular events $[1,2]$. The Japanese Society for the Study of Obesity also used the MetS criteria of abdominal circumference, blood pressure $(130 / 85)$, triglyceride $(150 \mathrm{mg} / \mathrm{dL})$ or HDL cholesterol $<40 \mathrm{mg} / \mathrm{dL}$ and blood glucose $(\geq 110$ $\mathrm{mg} / \mathrm{dL}$ ). In this metabolic status, cardiovascular 
event risk has been demonstrated to be higher with a hazard ratio of 1.70-1.86 in a general Japanese population [3, 4]. Diabetes and CKD were both significant risk factors for cardiovascular events in Japanese prognostic cohort study (J-ACCESS study) using myocardial perfusion imaging (MPI) $[5,6]$.

Subsequently, a J-ACCESS-2 study has been performed in Japan in patients with diabetes asymptomatic for coronary artery disease (CAD) $[7,8]$. There have been no studies in Japan dealing with asymptomatic patients with MetS and its relationship to cardiovascular events in conjunction with MPI. Our hypothesis in this study was that MetS might increase the risk of cardiovascular events even in patients with diabetes asymptomatic for CAD. The significance of perfusion abnormality in this study group was also examined.

\section{Methods}

\section{Subjects}

J-ACCESS-2 prognostic databases involving 513 patients from 50 institutions were used with a follow-up period of 3 years [7]. The criteria for registry included patients with type 2 diabetes, $\geq 50$ years of age, who either had maximal carotid artery intima-media thickness $\geq 1.1 \mathrm{~mm}$ by ultrasonography or a urinary albumin excretion rate of $\geq 30 \mathrm{mg} / \mathrm{g}$ creatinine, or patients who satisfied at least two of the following four conditions: abdominal obesity with body mass index (BMI) $\geq 25$ and waist circumference $\geq 85 \mathrm{~cm}$ for men and $\geq 90 \mathrm{~cm}$ for women, hypo-high density lipoprotein (HDL)-cholesterolemia $(<40 \mathrm{mg} / \mathrm{dl})$, hypertriglyceridemia $(\geq 150 \mathrm{mg} / \mathrm{dl})$, and hypertension (blood pressure $\geq 130 / 85 \mathrm{mmHg}$ ). Exclusion criteria included patients with myocardial infarction, effort angina, unstable angina, and those with abnormal electrocardiographic findings of ST depression $>1 \mathrm{~mm}$, multifocal premature ventricular contraction, tachyarrhythmia and atrial fibrillation. Also excluded were patients with hemoglobin A1c (HbA1c) $\geq 10 \%$ or serum creatinine $\geq 1.5 \mathrm{mg} / \mathrm{dl}$, valvular heart disease, idiopathic cardiomyopathy, New York Heart Association class III or IV heart failure at the time of MPI, and arteriosclerosis obliterans of the lower extremities (with intermittent claudication, resting pain and ulceration).

An institutional review board or committee approved the study in all institutions, and written informed consent was obtained from all subjects.

Definition of metabolic syndrome

In this subanalysis of the J-ACCESS-2 investigation, the criteria of MetS were determined by three or more of the following conditions: (1) $\mathrm{BMI} \geq 25 \mathrm{~kg} / \mathrm{m}^{2}$, (2) dyslipidemia (triglyceride $\geq 150 \mathrm{mg} / \mathrm{dL}$, HDL cholesterol $\leq 40 \mathrm{mg} / \mathrm{dL}$, or medication for dyslipidemia), (2) hypertension (systolic pressure $\geq 130 \mathrm{mmHg}$, diastolic pressure $\geq 85 \mathrm{mmHg}$ or medication for hypertension) and (3) diabetes (fasting blood sugar $\geq 110 \mathrm{mg} / \mathrm{dL}$, or medications or insulin therapy for diabetes). Abdominal circumference criteria were not used in this subanalysis [9].

\section{Myocardial perfusion imaging}

Stress-rest MPI was performed with ${ }^{99 \mathrm{~m}} \mathrm{Tc}-$ tetrofosmin. Exercise $(71 \%)$ or pharmacological stress (29\%) with dipyridamole, adenosine or adenosine triphosphate was performed. The average dose of $99 \mathrm{~m}$ Tc-tetrofosmin administered was $331 \mathrm{MBq}$ for the initial study and $748 \mathrm{MBq}$ for the second study. MPI data acquisition was performed with standard protocols, and a gated study was performed at least at resting condition. Myocardial images of short-axis, vertical long-axis and horizontal long-axis images were generated and interpreted using a 20-segment model. Segmental myocardial defects were visually scored using a 5-point scoring system: $0=$ normal, $1=$ mildly reduced, $2=$ moderately reduced, $3=$ severely reduced, and $4=$ absent. Total defect scores were calculated as the sum of stress, rest and difference scores (SSS, SRS, and SDS). Four SSS severity categories were used for risk-based groups of 0-3 (normal), 4-8 (mild), 9-13 (moderate), and $\geq 14$ (severe). Quantification of the gated SPECT was performed by QGS software (Cedars Sinai Medical Center, Los Angeles, CA, USA) at each institution. The left ventricular ejection fraction (LVEF, \%), end-diastolic volume (EDV, $\mathrm{ml}$ ), and end- systolic volume (ESV, ml) were calculated.

Coronary angiography was performed in 48 patients after MPI, and significant coronary artery stenosis was observed in 11 patients with MetS and 14 with non-MetS. The multi-vessel disease was observed in 4 patients with MetS and 8 with nonMetS ( $\mathrm{p}=0.93$ for number of coronary artery stenosis between MetS and non-MetS groups).

\section{Definition of events and follow-up}

The cardiovascular and cerebrovascular events were investigated each year after the registration. After excluding patients who had early revascularization within 30 days after registration $(\mathrm{n}=7)$, noncardiac death $(\mathrm{n}=7)$ and patients lost during follow-up ( $n=14)$, a total of 485 patients were selected for prognostic analysis. Events of cardiac death, sudden death (natural, rapid and unexpected death from cardiac causes: witnessed cardiac arrest or death $<1$ hour after the onset, or unwitnessed deaths within 24 hours after the patients were last seen to be alive), and nonfatal acute coronary syndrome (ACS) were defined as hard events. Total cardiovascular events further included percutaneous coronary intervention (PCI), 
coronary artery bypass grafting (CABG), de novo diagnosed stable angina, unstable angina and severe heart failure requiring hospitalization. Other vascular-related events such as transient ischemic attack of the brain, stroke, and de novo diagnosed peripheral vascular disease with intermittent claudication, resting pain and ulceration were also included in the total events.

\section{Statistics}

Continuous variables are expressed as mean \pm standard deviation (SD). We applied the Wilcoxon rank sum test to compare results from patients with and without cardiovascular events and the Chi-square test to categorical data. The univariate Cox proportional hazard model was used to evaluate each variable. The dependent variable included the occurrence of total events, cardiovascular events and death. The relative hazard ratios (HRs) and 95\% confidence of interval (CI) were calculated. The multivariate Cox proportional model was applied based on statistically significant independent variables. Kaplan-Meier analysis was also peformed to analyse survival curves. Statistical significance was defined as $\mathrm{p}<0.05$.

\section{Results}

Comparison of groups between MetS and nonMetS groups

Based on the definition of MetS, 229 patients were classified into the MetS group and 256 into the non-MetS group (Table 1). The MetS group showed higher BMI and larger abdominal circumference than the non-MetS group. With respect to dyslipidemia, HDL cholesterol was lower in the MetS group, but low-density lipoprotein (LDL) cholesterol was comparable between both groups. Gated SPECT showed that EDV and ESV were higher in the MetS group than in the nonMetS group. However, the degree of ischemia as determined by SSS or SDS and resting perfusion defect by SRS was similar between MetS ans nonMetS groups. During the follow up of three years, 14 cardiovascular hard events occurred. The event rate of cardiac death and cardiovascular events did not differ significantly between the two groups. When the breakdown of total events other than death was observed, cardiac disease related events totaled 11 for MetS and 19 for non-MetS groups, cerebrovascular events numbered 15 for MetS and 6 for non-MetS groups, and peripheral vascular disease related events totaled 3 for MetS and 3 for non-MetS groups (Pearson test, $\mathrm{p}=0.103$ ).

\section{Cox proportional hazard analysis}

Based on univariate Cox proportional hazard analysis, significant variables related to total events included age, current smoking, insulin use, total cholesterol, LVEF, SSS $\geq 9$ and SDS $\geq 2$ (Table 2). However, to predict cardiovascular hard events, only insulin and urea nitrogen were significant, and SSS, SDS and eGFR were not significant. In the hard events, the multivariate analysis was not accepted because of a relatively low incidence of hard events. In the total events, multivariate analysis showed no additional variables to the SSS $\geq 9$. Coronary arter stenosis documented during the course of follow-up was analyzed by the univariate analysis. The hazard ratios of the presence of coronary artery stenosis for total events were $4.63(\mathrm{p}<0.0001)$, and that for cardiovascular hard events was $3.57(\mathrm{p}=0.096)$.

\section{MetS as a predictor of events}

To evaluate the role of variables related to MetS, the presence of MetS and the number of items for MetS were included for Cox proportional hazard analysis (Table 3). However, the variables of MetS were not significantly related to cardiac death and total events. In contrast, SSS $\geq 9$ and $\mathrm{SDS} \geq 2$ were significantly related to total events $(\mathrm{HR}=2.32$ for $\mathrm{SSS}$ and 3.45 for SDS).

\section{Differences in medication}

When the MetS and non-MetS groups were compared, the MetS group had higher incidences of biguanide $(23.1 \%$ vs. $14.1 \%, \mathrm{p}<0.0138)$, angiotensin II antagonist $(45.1 \%$ vs. $30.1 \%$, $0.0010)$, calcium channel blocker $(53.7 \%$ vs. $32.8 \%, \mathrm{p}<0.0001)$, alfa blocker $(8.3 \%$ vs. $2.3 \%$, $\mathrm{p}=0.0059)$, and lower incidence of aspirin $(15.7 \%$ vs. $25.8 \%, \mathrm{p}=0.0093)$. No statistical differences were found between the two groups in terms of medication of sulfonyluria $(47.2 \%$ vs. $43.0 \%$, $\mathrm{p}=0.40)$, statin $(38.4 \%$ vs. $31.6 \%, \mathrm{p}=0.14)$ and insulin use (27.1\% vs. $32.4 \%, \mathrm{p}=0.24)$.

\section{Survival analysis}

Kaplan-Meier survival analysis was performed in patients with SSS $\leq 9$ and $<9$, and with and without MetS (Figure 1). When all events were analyzed, the SSS $\geq 9$ group showed higher incidence of total events, but cardiovascular events including cardiac death, sudden death and ACS did not reach statistical significance when both groups were compared. Patients with and without MetS did not show significant differences in total event analysis. When patients were classified based on SSS and MetS, the groups differed significantly. However the contribution of SSS was more significant as shown in Figure 1D.

\section{Discussion}

MetS is an important predictor of cardiovascular events. In asymptomatic patients with diabetes, however, the effects of additional factors of MetS on cardiovascular events have not 
been clarified. In this subanalysis based on JACCESS-2 databases, we classified patients into MetS and non-MetS groups and found that the only factor related to total cardiovascular events was myocardial perfusion abnormality at stress or ischemia, and the MetS was not the significant predictor in this study population. Cardiovascular events and ischemia were commonly observed in diabetic patients without MetS as in the case in those with MetS.

The J-ACCESS-2 investigation was a multicenter prognostic investigation involving asymptomatic type-2 diabetes [8]. The selection criteria included abdominal circumference, BMI, hypertension and dyslipidemia. The MetS criteria were satisfied in approximately half of the asymptomatic patients. The total event rate was observed in $13 \%$ of the patients, although the cardiac death rate was relatively small. Multivariate Cox proportional hazard analysis showed that significant variables for total cardiovascular events comprised stress perfusion defect ( $\mathrm{SSS} \geq 9$ ), a low eGFR, and being a current smoker. We therefore tried to clarify the contribution of MetS to occurrence of cardiovascular events, since a noninvasive stress test was sometimes indicated even in asymptomatic populations of MetS. However, although the MetS group showed a higher incidence of abnormalities for dyslipidemia, hypertension and eGFR as well as large ventricular volumes than the non-MetS group, no difference was observed between the two groups with respect to total and hard cardiovascular event rates.

There was no difference in any of the MPI variables between the MetS and no Mets groups. This was probably caused by the therapeutic effects for ischemia including PCI and medication, which resulted in reduction of hard cardiovascular events. It should be noted that abnormality of MPI was related to total events including cerebrovascular events and peripheral vascular events. MPI abnormality cannot directly be used as a predictor for non-cardiac events. However, cerebrovascular events are the third highest cause of mortality in Japan, and factors of diabetes, dyslipidemia and hypertension, in addition to an aging society, are major backgrounds commonly found related to the total events in this study. Considering these metabolic factors, the higher total event rate in patients with abnormal MPI might be explained.

A study in the United States using 7,849 patients from a multicenter prospective registry analyzed the importance of MetS for predicting cardiovascular events [10]. There was an additive value in predicting major cardiac events using the number of risk factors for MetS and the extent and severity of abnormalities in MPI. Patients with five risk factors of MetS were of particularly high risk showing hazard ratios from 7.8 to 14.1 -fold for mild to severely abnormal MPI findings. Acampa et al. showed that in a IDIS multicenter prospective study in diabetec patients, addition of MPI data to a prediction model based on traditional risk factors and ECG stress test data significantly improved coronary heart disease risk classification [11]. In a Japanese study involving 1,424 type-2 diabetic patients, a high prevalence (38-53\%, depending on sex and definition) of MetS was found among diabetic patients [12]. Although coronary heart disease events were observed in $4.4 \%$ during an 8year study, the individual components of MetS, such as hyperlipidemia or hypertension, were equivalent or better predictors than the definition of MetS in men. The present study did not confirm the significance of MetS for major cardiovascular events. This was probably due to selection bias during the J-ACCESS-2 investigation, since cardiac death and cardiovascular hard event rates were in $1.2 \%$ and $2.9 \%$ per 3 years, respectively. Another factor for contributing to the equivalent event rate was the effect of more active medications in the MetS group, which presumably improved their metabolic status and reduced mortality. Therefore, in the group with a low-risk profile for coronary artery disease and medically treated patients as in the J-ACCESS-2 study, MetS was not the major predictor of cardiac events.

This subanalysis was performed to see whether the presence of MetS had siginifcant effects on screening in patients with type 2 diabetes. The effectiveness of screening by MPI in asymptomatic diabetic patients was investigated in a DIAD-2 study [13]. It was found that cardiac event rates were low and were not significantly reduced by MPI screening for myocardial ischemia over a 4.8 year period. However, the positive predictive value of having moderate or large MPI defects was $12 \%$. Although screening all patients with diabetes is not justified, restricting screening to a patient population in which a relatively high positive yield can be ensured would be a practical approach. [14, 15]. In fact the factor of $\mathrm{SSS} \geq 9$ showed high total event rate in this study. Based on the MPI stress abnormality, optimal medical therapy and/or percutaneous coronary intervention would be indicated for those patients, which might subsequently result in improved patient outcome. The selection criteria for further non-invasive stress testing might include typical chest symptoms, electrocardiographic abnormalities with treadmill exercise, high coronary calcium scoring, etc. as evidenced by nuclear cardiology guidelines in the USA and Japan [16, 17]. Coronary artery calcium scoring was not included in this study. Some of the patients with minimal or absent coronary calcification might have been excluded, and those 
with increased atherosclerotic burden would have been indicated for MPI studies [18]. Since the impact of MPI and MetS may also differ among ethnic backgrounds, an optimal test in symptomatic and non-symptomatic patients for CAD should be further investigated in various populations.

Limitations. A larger number of subjects or a longer follow-up period are required to better analyze the hard cardiac events. Since only asymptomatic patients were included in this study, a comparative risk group study may be indicated by including symptomatic patients or those with moderate likelihood of coronary artery disease. The selection criteria for MetS used $\mathrm{BMI} \geq 25$, which was lower than the criteria of WHO using $\geq 30$, but it has been noted that Japanese patients with type 2 diabetes are not obese compared with American and some European poulations [19]. Although patients with type-1 diabetes appear to have greater risk of cardiac death than other patients based on any MPI result, we included type- 2 diabetes in this study [20].

\section{Conclusion}

In patients with type 2 diabetes asymptomatic for CAD, the presence of MetS did not increase the rate of hard and total cardiac events. Cardiovascular events are observed in diabetic patients without MetS similar to those with MetS. However, moderate to severe SSS $\geq 9$ showed high incidence of total cardiovascular events. Therefore, a routine screening test by MPI for asymptomatic patients having MetS criteria is not indicated, but potentially higher-risk population should be further investigated.

\section{Conflict of interst: none}

Acknowledgement This work was mainly supported by the Japan Cardiovascular Research Foundation, and in part by JSPS Grants-in-Aid for Scientific Research (C) Grant Number 22591320 in Japan. We thank Mr. Ronald Belisle for editorial assistance.

\section{References.}

1. Definition, Diagnosis and Classification of Diabetes Mellitus and its Complications. Report of a WHO Consultation. Part 1: Diagnosis and Classification of Diabetes Mellitus. World Health Organization Department of Noncommunicable Disease Surveillance, Geneva. . 1999.

2. Executive Summary of The Third Report of The National Cholesterol Education Program (NCEP) Expert Panel on Detection, Evaluation, And Treatment of High Blood Cholesterol In Adults (Adult Treatment Panel III). JAMA. 2001;285:2486-97.

3. Ninomiya T, Kubo M, Doi Y, Yonemoto K, Tanizaki Y, Rahman M, et al. Impact of metabolic syndrome on the development of cardiovascular disease in a general Japanese population: the Hisayama study. Stroke. 2007;38:2063-9.

4. Ninomiya $\mathrm{T}$, Kiyohara $\mathrm{Y}$, Kubo $\mathrm{M}$, Yonemoto K, Tanizaki Y, Doi Y, et al. Metabolic syndrome and CKD in a general Japanese population: the Hisayama Study. Am J Kidney Dis. 2006;48:383-91.

5. Nakajima K, Matsuo S, Okuyama C, Hatta T, Tsukamoto K, Nishimura S, et al. Cardiac event risk in Japanese subjects estimated using gated myocardial perfusion imaging, in conjunction with diabetes mellitus and chronic kidney disease. Circ J. 2012;76:168-75.

6. Nishimura T, Nakajima K, Kusuoka $H$, Yamashina A, Nishimura S. Prognostic study of risk stratification among Japanese patients with ischemic heart disease using gated myocardial perfusion SPECT: J-ACCESS study. Eur J Nucl Med Mol Imaging. 2008;35:319-28.

7. Yamasaki $\mathrm{Y}$, Nakajima K, Kusuoka $\mathrm{H}$, Izumi $\mathrm{T}$, Kashiwagi A, Kawamori $\mathrm{R}$, et al. Prognostic value of gated myocardial perfusion imaging for asymptomatic patients with type 2 diabetes: the J-ACCESS 2 investigation. Diabetes Care. 2010;33:2320-6.

8. Nakajima K, Yamasaki Y, Kusuoka H, Izumi T, Kashiwagi A, Kawamori $\mathrm{R}$, et al. Cardiovascular events in Japanese asymptomatic patients with type 2 diabetes: a 1-year interim report of a J-ACCESS 2 investigation using myocardial perfusion imaging. Eur J Nucl Med Mol Imaging. 2009.

9. Doi Y, Ninomiya T, Hata J, Yonemoto K, Arima H, Kubo M, et al. Proposed criteria for metabolic syndrome in Japanese based on prospective evidence: the Hisayama study. Stroke. 2009;40:1187-94.

10. Shaw LJ, Berman DS, Hendel RC, Alazraki N, Krawczynska E, Borges-Neto S, et al. Cardiovascular disease risk stratification with stress single-photon emission computed tomography technetium- $99 \mathrm{~m}$ tetrofosmin imaging in patients with the metabolic syndrome and diabetes mellitus. Am J Cardiol. 2006;97:1538-44.

11. Acampa W, Petretta M, Evangelista L, Daniele S, Xhoxhi E, De Rimini ML, et al. Myocardial perfusion imaging and risk classification for coronary heart disease in diabetic patients. The IDIS study: a prospective, multicentre trial. Eur J Nucl Med Mol Imaging. 2012;39:387-95.

12. Sone H, Mizuno S, Fujii H, Yoshimura Y, Yamasaki Y, Ishibashi S, et al. Is the diagnosis of metabolic syndrome useful for predicting cardiovascular disease in asian diabetic patients? Analysis from the Japan Diabetes Complications Study. Diabetes Care. 2005;28:1463-71.

13. Young LH, Wackers FJ, Chyun DA, Davey JA, Barrett EJ, Taillefer R, et al. Cardiac outcomes 
after screening for asymptomatic coronary artery disease in patients with type 2 diabetes: the DIAD study: a randomized controlled trial. JAMA. 2009;301:1547-55.

14. Wackers FJ. Asymptomatic patients with diabetes mellitus should be screened for coronary artery disease. J Nucl Cardiol. 2006;13:609-15.

15. Gibbons RJ. Asymptomatic patients with diabetes mellitus should not be screened for coronary artery disease. J Nucl Cardiol. 2006;13:616-20.

16. Klocke FJ, Baird MG, Lorell BH, Bateman TM, Messer JV, Berman DS, et al. ACC/AHA/ ASNC guidelines for the clinical use of cardiac radionuclide imaging--executive summary: a report of the American College of Cardiology/American Heart Association Task Force on Practice Guidelines (ACC/AHA/ASNC Committee to Revise the 1995 Guidelines for the Clinical Use of Cardiac Radionuclide Imaging). Circulation. 2003;108:1404-18.
17. Yamashina A. Guidelines for Noninvasive Diagnosis of Coronary Artery Lesions (JCS 2009). Circ J. 2009;73 (Suppl III):1019-89.

18. Anand DV, Lim E, Hopkins D, Corder R, Shaw LJ, Sharp P, et al. Risk stratification in uncomplicated type 2 diabetes: prospective evaluation of the combined use of coronary artery calcium imaging and selective myocardial perfusion scintigraphy. Eur Heart J. 2006;27:713-21.

19. Sone H, Yoshimura Y, Ito H, Ohashi Y, Yamada N. Energy intake and obesity in Japanese patients with type 2 diabetes. Lancet. 2004;363:248-9.

20. Robich MP, Osipov RM, Chu LM, Han Y, Feng J, Nezafat R, et al. Resveratrol modifies risk factors for coronary artery disease in swine with metabolic syndrome and myocardial ischemia. Eur J Pharmacol. 2011;664:45-53. 
Table 1. Comparison of patient characteristics between MetS and non-MetS groups

\begin{tabular}{|c|c|c|c|}
\hline & MetS $(n=229)$ & Non-MetS $(\mathrm{n}=256)$ & $\mathrm{p}$ \\
\hline$\overline{\text { Age (years) }}$ & $67 \pm 7$ & $67 \pm 7$ & 0.23 \\
\hline Male gender (\%) & $53 \%$ & $62 \%$ & 0.07 \\
\hline Body mass index $\left(\mathrm{kg} / \mathrm{m}^{2}\right)$ & $27.7 \pm 2.7$ & $22.1 \pm 1.9$ & $<.0001$ \\
\hline Abdominal circumference $(\mathrm{cm})$ & $92.5 \pm 10.2$ & $81.2 \pm 7.6$ & $<.0001$ \\
\hline Max IMT (mm) & $1.53 \pm 0.69$ & $1.73 \pm 0.87$ & 0.06 \\
\hline Hypertension & $90.8 \%$ & $75.8 \%$ & $<.0001$ \\
\hline Dyslipidemia & $84.7 \%$ & $75.4 \%$ & 0.02 \\
\hline HDL cholesterol (mg/dL) & $48.7 \pm 13.4$ & $53.1 \pm 15.5$ & 0.01 \\
\hline LDL cholesterol (mg/dL) & $120.0 \pm 29.4$ & $115.2 \pm 32.6$ & 0.12 \\
\hline Triglyceride (mg/dL) & $184.0 \pm 97.5$ & $154.8 \pm 127.1$ & $<.0001$ \\
\hline Insulin & $27.1 \%$ & $32.4 \%$ & 0.24 \\
\hline Statin & $34.4 \%$ & $31.6 \%$ & 0.14 \\
\hline Creatinine (mg/dL) & $0.81 \pm 0.25$ & $0.77 \pm 0.23$ & 0.06 \\
\hline eGFR (ml/min/1.73m²) & $70.4 \pm 20.5$ & $74.9 \pm 19.4$ & 0.02 \\
\hline Urea Nitrogen (mg/dL) & $17.1 \pm 5.1$ & $16.6 \pm 5.0$ & 0.33 \\
\hline Urinary albumin $(\log )$ & $3.97 \pm 1.44$ & $3.93 \pm 1.40$ & 0.94 \\
\hline \multicolumn{4}{|l|}{ Gated SPECT analysis (QGS) } \\
\hline $\mathrm{EDV}(\mathrm{mL})$ & $77.1 \pm 23.0$ & $70.5 \pm 20.7$ & 0.003 \\
\hline ESV (mL) & $26.8 \pm 13.9$ & $23.7 \pm 12.2$ & 0.01 \\
\hline $\mathrm{EF}(\%)$ & $66.7 \pm 9.6$ & $67.9 \pm 9.9$ & 0.38 \\
\hline \multicolumn{4}{|l|}{ Summed score by MPI } \\
\hline SSS & $2.92 \pm 4.64$ & $2.77 \pm 4.55$ & 0.29 \\
\hline SRS & $2.03 \pm 3.65$ & $2.23 \pm 3.77$ & 0.89 \\
\hline SDS & $0.88 \pm 3.25$ & $0.54 \pm 2.33$ & 0.65 \\
\hline \multicolumn{4}{|l|}{ Cardiovascular events } \\
\hline Death & $2(0.9 \%)$ & $3(1.2 \%)$ & 0.78 \\
\hline Cardiovascular hard events & $6(2.6 \%)$ & $8(3.1 \%)$ & 0.95 \\
\hline Total events & $31(13.5 \%)$ & $31(12.1 \%)$ & 0.74 \\
\hline
\end{tabular}

IMT, intima-media thickness; MPI, myocardial perfusion imaging; EDV, end-diastolic volume; ESV, endsystolic volume; EF, ejection fraction; SSS, summed stress score; SRS, summed rest score; SDS, summed difference score 
Table 2 Univariate Cox analysis for total and hard cardiovascular events

\begin{tabular}{|c|c|c|c|c|c|c|}
\hline Variables & Total events & $\begin{array}{c}\text { Hazard } \\
\text { Ratio }\end{array}$ & $\mathrm{p}$ & $\begin{array}{l}\text { Cardio- } \\
\text { vascular hard } \\
\text { events } \\
\text { Wald } \chi^{2}\end{array}$ & $\begin{array}{c}\text { Hazard } \\
\text { Ratio }\end{array}$ & $\mathrm{p}$ \\
\hline Age & 4.66 & 1.04 & 0.03 & 1.40 & 1.04 & 0.24 \\
\hline Sex & 0.004 & 0.98 & 0.95 & 0.25 & 0.76 & 0.62 \\
\hline Body mass index & 1.25 & 0.96 & 0.26 & 3.64 & 0.85 & 0.06 \\
\hline Family history of CAD & 0.03 & 0.90 & 0.86 & 0.0001 & 0.00 & 0.99 \\
\hline Current smoking & 4.29 & 1.85 & 0.04 & 0.01 & 1.10 & 0.91 \\
\hline Hypertension & 3.59 & 2.42 & 0.06 & 0.21 & 0.75 & 0.65 \\
\hline Dyslipidemia & 0.28 & 0.85 & 0.60 & 0.01 & 0.92 & 0.90 \\
\hline Insulin use & 5.03 & 1.78 & 0.03 & 4.66 & 3.21 & 0.03 \\
\hline Medication of statin & 3.54 & 0.57 & 0.06 & 2.36 & 0.31 & 0.12 \\
\hline ECG abnormality at rest & 3.03 & 1.68 & 0.08 & 1.19 & 1.95 & 0.28 \\
\hline Total cholesterol & 4.17 & 1.01 & 0.04 & 0.52 & 1.01 & 0.47 \\
\hline LDL-cholesterol & 2.05 & 1.01 & 0.15 & 1.10 & 1.01 & 0.30 \\
\hline HDL-cholesterol & 0.07 & 1.00 & 0.79 & 0.01 & 1.00 & 0.92 \\
\hline Triglyceride & 0.23 & 1.00 & 0.63 & 0.53 & 1.00 & 0.47 \\
\hline Fasting blood sugar & 3.56 & 1.00 & 0.06 & 3.78 & 1.01 & 0.05 \\
\hline Hemoglobin $\mathrm{A}_{1 \mathrm{C}}(\%)$ & 1.41 & 0.88 & 0.23 & 0.03 & 0.96 & 0.85 \\
\hline Urea nitrogen & 9.18 & 1.06 & 0.002 & 4.28 & 1.09 & 0.04 \\
\hline Creatinine & 2.12 & 2.11 & 0.14 & 1.19 & 3.33 & 0.27 \\
\hline Urinary microalbumin & 0.39 & 1.00 & 0.53 & 0.10 & 1.00 & 0.75 \\
\hline LVEF & 5.13 & 0.97 & 0.02 & 2.10 & 0.96 & 0.15 \\
\hline EDV & 0.001 & 1.00 & 0.97 & 0.24 & 0.99 & 0.63 \\
\hline ESV & 1.25 & 1.01 & 0.26 & 0.12 & 1.01 & 0.73 \\
\hline SSS & 2.51 & 1.04 & 0.11 & 0.71 & 1.04 & 0.40 \\
\hline SRS & 0.39 & 1.02 & 0.53 & 0.89 & 1.05 & 0.34 \\
\hline SDS & 3.21 & 1.06 & 0.07 & 0.01 & 1.01 & 0.90 \\
\hline Abdominal circumference & 0.02 & 1.00 & 0.89 & 0.01 & 1.00 & 0.92 \\
\hline MaxIMT & 0.00 & 1.01 & 0.97 & 0.04 & 1.14 & 0.84 \\
\hline eGFR & 2.34 & 0.99 & 0.13 & 0.67 & 0.99 & 0.41 \\
\hline
\end{tabular}

CAD, coronary artery disease; ECG, electrocardiogrm; EDV, end-diastolic volume; ESV, endsystolic volume; EF, ejection fraction; SSS, summed stress score; SRS, summed rest score; SDS, summed difference score; IMT, intima-media thickness; 
Table 3 Univariate Cox analysis for selected variables

\begin{tabular}{lccccc}
\hline & Wald $\chi^{2}$ & $\begin{array}{c}\text { Hazard } \\
\text { Ratio }\end{array}$ & $\begin{array}{c}95 \% \text { CI } \\
\text { Lower }\end{array}$ & $\begin{array}{c}\text { 95\% CI } \\
\text { Upper }\end{array}$ & $p$ \\
\hline Total events & & & & & \\
Criteria of MetS & 0.17 & 1.11 & 0.67 & 1.82 & 0.68 \\
Number of items for MetS & 0.33 & 1.16 & 0.70 & 1.93 & 0.56 \\
eGFR $\geq 60,<60$ & 1.56 & 1.42 & 0.82 & 2.45 & 0.21 \\
SSS $\geq 9,<9$ & 6.42 & 2.32 & 1.21 & 4.46 & 0.011 \\
SDS $\geq 2,<2$ & 5.73 & 3.45 & 1.25 & 9.51 & 0.017 \\
Cardiovascular hard events & & & & & \\
Criteria of MetS & 0.11 & 0.84 & 0.29 & 2.41 & 0.74 \\
Number of items for MetS & 0.49 & 1.53 & 0.47 & 5.00 & 0.49 \\
eGFR $\geq 60,<60$ & 0.66 & 1.66 & 0.49 & 5.67 & 0.42 \\
SSS $\geq 9,<9$ & 2.16 & 2.61 & 0.73 & 9.34 & 0.14 \\
SDS $\geq 2,<2$ & 1.25 & 3.19 & 0.42 & 24.35 & 0.26 \\
\hline
\end{tabular}

A. Total events in patients regarding SSS groups

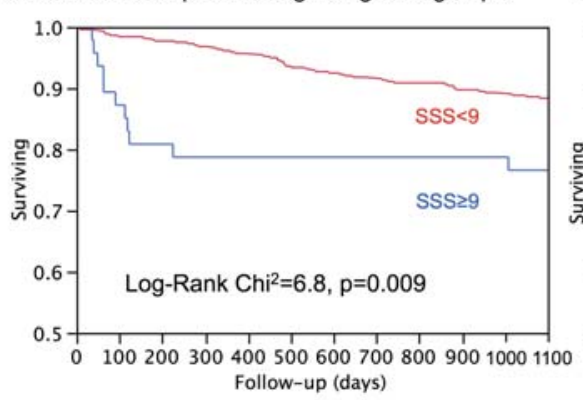

C. Total events regarding MetS groups

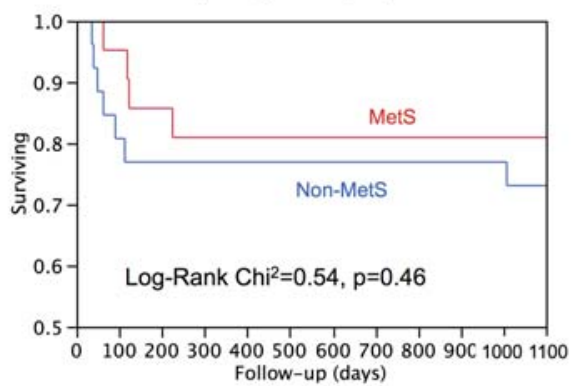

B. Cardiac hard events regarding SSS groups

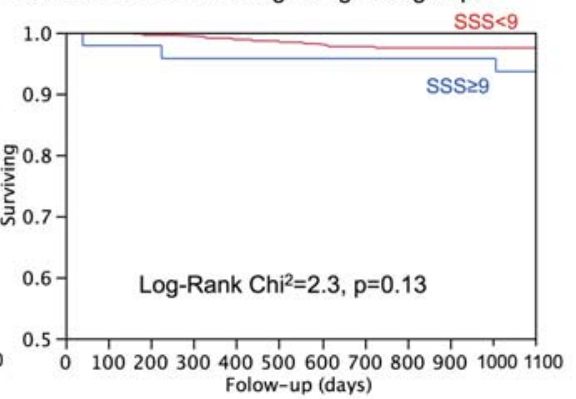

D. Total events regarding SSS and MetS groups

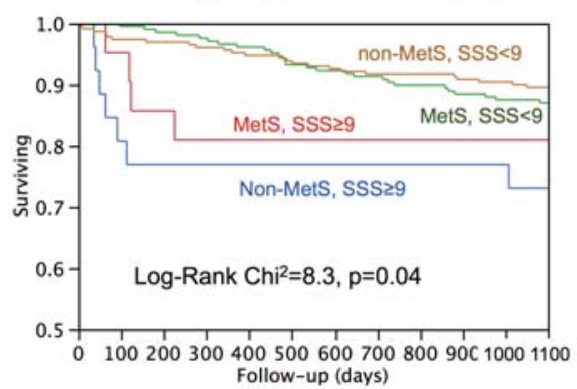

Figure 1

Kaplan-Meier survival analyses for the MetS and non-MetS groups and SSS values. Higher SSS has significant impact on the total event rate, but not on mortality. 